\title{
A biological melange and a peculiar led light could be a thaumaturgic approach to defeat manifold types of folliculitis in man and woman
}

\section{Lorenzo Martini}

University of Siena, Department of Pharmaceutical Biotechnologies, Via A. Moro 2, 53100 Siena, Italy

Corresponding author: Lorenzo Martini, M.Sc., E-mail: martinil163@libero.it

\begin{abstract}
Nowadays PTD (photodynamic therapy) together with methyl aminolevulinate (MAL) pomades seem to be the goal and merit of all the vanguard dermatologists and aesthetic surgeons, who are determined to struggle radically the problems of folliculitis, malaise that disturbs men and women, even by the social point of view. In this study We attempt to treat 12 different cases of folliculitis caused by symptoms of different etiologies in men, women and in a transvestite, but not operate (idest a pre-op trans), using a cosmetic formula made of a particular fermented rice starch and particular irradiation for different quantities of time, depending on the gravity of the disease to treat. Results are utterly satisfying.
\end{abstract}

Key words: PTD; Folliculitis; LED; MAL; Protoporphirin IX

\section{INTRODUCTION}

Folliculitis is an inflammation of the hair follicles. Folliculitis may occur onto any part of human body that has hair. But it is most common on the beard area, arms, back, buttocks, inner thighs and legs and can be caused by bacteria, howbeit it also can be caused by yeast or another type of fungi.

Anyway its manifestation is always accompanied by erythema and harassing itch.

Folliculitis could even arise owing to damaged hair follicles. Shaving, depilating, epilating or wearing habiliment that rubs the skin can irritate the follicles, which can lead to folliculitis. They also can become blocked or irritated by sweat, machine oils, or makeup. When the follicles are injured, they are more likely to become infected.

For, folliculitis is common in men and women as well, and symptoms are very fastidious and disagreeable.

Dermatologists too often suggest the continuous usage of corticosteroids or Mupirocin lipogels and aqueous lotions and an avalanche of natural remedies exist, as the ancient "Venus' milk", based on benzoin resin oil dispersed in rose water, (notwithstandind its way of action is almost unclear, it appears to be the most preferable choice advised by herbalists) and/or sanative hydrolats based on brimstone salts and its derivatives.

When folliculitis is evoked by a serious fungal infection, as Malassezia folliculitis, oral antifungal medications are favoured, however antifungal remedies too often show many therapeutic weaknesses such as infection relapse, drug resistance, or adverse effects like hepatotoxicity and gastrointestinal discomfort [1].

Hereupon, we have decided to treat severe cases of recalcitrant folliculitis by a sort of PDT (photodynamic therapy), as divers dermatologists nowadays rede as an alternative therapeutic option for its antimicrobial effect, and we have chosen a type of revisited method of PDT.

Forsooth, PDT forecasts the use of methyl aminolevulinate (MAL) cream together with

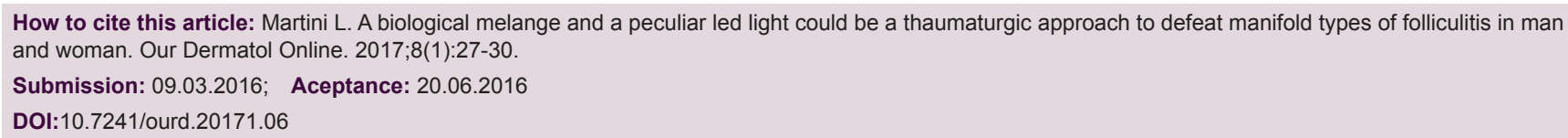


irradiations emitted from a simplest lamp with lightemitting diodes (LED), easily available on the market.

MAL is considered an optimal sensitizer in photodynamic therapy, since it acts as a prodrug that is metabolized to protoporphyrin IX.

Now, Protoporphyrin IX is an important precursor to biologically essential prosthetic groups such as cytochrome $\mathrm{C}$ in mammals and chlorophylls in herbs. As a result, a number of organisms are able to synthesize Protoporphyrin IX from basic precursors such as glycine and succinyl CoA, or glutamate [2-4].

Glutamate occurs naturally in many foods, such as tomatoes and cheeses and in a major extent in Kelp, seaweeds and fermented starch (especially from cassava roots), sugar beets, sugar cane or molasses by the aids of Aspergillus spp.

Since today, instead of extracting and crystallizing glutamate from seaweed broth, it is preferable to produce it by the fermentation of manifold starches (and this fermentation process is similar to that used to make yogurt, vinegar and wine) we have decided to arrange a type of fermented starch following a revolutionary and nonconventional method heralded by Seinosuke et al [5] and thus letting to drive a complete fermentation of rice starch and soybean homogenate-enzymes-yeast mixture in a cruse at pH 3.5 and 545.67 Rankine degrees for five days, to obtain a surrogate of a fermented starch, that is admitted as ASPERGILLUS/GLUCOSE/SOYBEAN/ STARCH FERMENT FILTRATE in the International Nomenclature Cosmetic Index.

The irradiation is provided by a simplest LED lamp, capable to emit light radiations at $630 \mathrm{~nm}$.

The times of exposure to irradiation is calculated on the basis of the intensity and severity of the folliculitis itself, and so, for all cases where the bacterial assault is the only responsible (confiding on the dermatologist's judgement or in same extreme case on the histological report, when available) 10 minutes/day of exposure is suggested for 2 weeks, while in all cases which by the Satter's method (the $\mathrm{KOH}$ inspection) is possible to detect a fungal infection, 20 minutes of irradiation pro day are suggested $[6,7]$.

Satter's method (idest the KOH inspection) is based on the deposition of a sample (scare or papula picked up from the erythematous area of the folliculitis) on a slide where a standardized $\mathrm{KOH}$ solution is placed in order to dissolve all the non-fungal elements, and thus by a simple examination by electronic microscope yeast cells and fungal hyphae (branching filaments) are detectable easily and an eventual gradual regression may be recorded, during and after treatment.

\section{MATERIALS AND METHODS}

We have recruited 12 volunteers, and thus:

2 (A and B) young men presenting severe folliculitis due to beard shaving.

1 (C) middle age man presenting a mild folliculitis due to beard shaving.

1 (D) streapteaseuse, who must have all legs epilated (it must be kept on account that she loves to have suppers consuming shellfishes and molluscs, food extremely rich in haemocyanins, which are photosensitizing agents).

2 ( $\mathrm{E}$ and $\mathrm{F}$ ) girls who love to depilate their inguinal areas.

1) (G) female clown endowed by a very delicate skin, suffering from folliculitis evoked by excessive circus make up.

2 (H and I) ripped men who like to epilate their chest, torso and axillae (it must be kept on account that Case $\mathrm{H}$ takes high dosages of nalidixic acid, that is a photosensitizing drug).

1 (L) transvestite who uses to epilate deeply all his body parts.

1 (M) classic dancer (young man) presenting a severe folliculits on his buttocks cause of his ballet habillement

$\mathrm{l}(\mathrm{N})$ middle age woman suffering from hirsutism and obliged to depilate twice a week her hirci of her axillae.

On the basis of their dermatological, histological or Satter's tests results I have traced an outline forecasting the following scores:

B1-B5 (indicating the degree of severity of the bacterial folliculitis). 
F1-F5 (indicating the degree of severity of the fungal infection, determined by the Satter's method) and thus I drawn Table 1, where even the minutes of irradiation suggested are plotted:

The cosmetic system "ASPERGILLUS/GLUCOSE/ SOYBEAN/STARCH FERMENT FILTRATE" to be spread onto the areas affected by folliculitis, was prepared by letting to drive a complete fermentation starting from a aqueous solution of rice starch together with soybean enzymes and Aspergillus niger in a cruse at $\mathrm{pH} 3.5$ (by lactic acid) and 545.67 Rankine degrees for five days.

The final cosmetic item is a colourless and odourless filtrate to be spread directly onto the areas of the body that manifest rashes of folliculitis and are then destined to be irradiated by a $100 \mathrm{~W}$ Watt Red High Power LED Light Lamp Plant Grow Growth 630nm. (Aktilite CL128), for the time requested for each of every specific case, following the values plotted in Table 1.

\section{RESULTS}

To collect results and to have the chance to demonstrate the efficacy of the treatment, the way I have chosen is the one that monitors the skin reflectance spectra using a normal spectrometer ranging from violet to green light, that is capable to determine the presence of the epidermal blood, by evaluating its intrinsic and peculiar absorbance.

Now, it is clear that blood excess, revealed by a certain type of spectrum, must be considered as an evident sign of erythema, on the other hand blood paucity, revealed by another type of spectrum, must be considered as an evident index of regression of the erythema.

To precisely determinate the general collapse of the erythematous rash in every case, I have exploited the Nielsen's method (The Norwegian Academy of Science and Letters, Oslo, 2008) [8,9], that keeps on account the main blood absorption bands that can be registered between the wavelengths 400 and $425 \mathrm{~nm}$ (violet light) and the wavelengths 500 and $600 \mathrm{~nm}$ (green light), and bands are registered as percent reflectivities.

By this way the usage of a simple spectrometer is sufficient to individuate how the different spectra recorded give information of the decrease of the epidermal blood flux, indicating the complete regression of the erythematous state of the skin itself.
The percent reflectivity (from 0.3 to $1.2 \%$ ) may indicate the scarceness or the plenitude of superficial blood on epidermis, and thus each case is represented by a value expressed as percentage, from 0.3 to 1.2 .

Low values (from 0.3 to 0.6 ) signify a dramatic decrement of the erythema, meanwhile high values (from 0.7 to 1.2 ) signify the presence of a frank erythema.

In Table 2 the initial and final scores (after treatment) are plotted.

It can be asserted that in every case a dramatic lowering of the symptoms of folliculitis (evidenced as collapse of skin erythematous manifenstations) is really achieved, apart from two cases that are discussed in the Conclusions.

\section{CONCLUSIONS}

All the cases I have cosmetically treated presented, at the very beginning of the experimentation, divers

Table 1: Time and minutes of irradiation forecasted for each of every case, depending on the severity of the folliculitis

\begin{tabular}{lcc}
$\begin{array}{l}\text { Case } \\
\text { number }\end{array}$ & $\begin{array}{c}\text { Type and degree } \\
\text { of folliculitis }\end{array}$ & $\begin{array}{c}\text { Time in minutes of irradiation } \\
\text { applied in the experimentation }\end{array}$ \\
\hline A & B3 & 10 \\
B & B5 & 10 \\
C & B1 & 10 \\
D & F2 & 20 \\
E & F3 & 20 \\
F & F4 & 20 \\
G & F2 & 20 \\
H & B3 & 10 \\
I & F1 & 20 \\
L & F5 & 20 \\
M & F3 & 20 \\
$N$ & B4 & 10 \\
\hline
\end{tabular}

Table 2: The values of the percent reflectivities of volunteers' skin, at the beginning and at the end of the treatment with "ASPERGILLUS/ GLUCOSE/SOYBEAN/STARCH FERMENT FILTRATE” and light

\begin{tabular}{lcc}
\hline Case & Initial percent reflectivity & Final percent reflectivity \\
\hline A & 0.7 & 0.3 \\
B & 0.6 & 0.5 \\
C & 0.8 & 0.4 \\
D & 0.9 & 0.7 \\
E & 1.1 & 0.5 \\
F & 1.2 & 0.4 \\
G & 1.0 & 0.3 \\
H & 0.9 & 0.8 \\
I & 0.7 & 0.4 \\
L & 0.9 & 0.6 \\
M & 1.1 & 0.3 \\
N & 1.0 & 0.4 \\
\hline
\end{tabular}


clinical pictures, regarding the presence of eventual bacterial or fungal infection and even the divers importance of the disease itself: anyway every case presented from mild to severe degrees of erythematous rashes, and this fact has elicited the possibility to calculate the real degree of regression of erythema (rubor) and thus the regression of the folliculitis as well.

It should be interesting to include even the skin colour of each individual as reference to better comprehend the real efficacy of this cosmetic treatment.

Anyway volunteers I have chosen are all white.

In a future it could be mandatory to evaluate eventual differences treating Asian or African individuals too.

It is remarkable that Case D, who likes to savour photosensitizing fishes and Case $\mathrm{H}$, who uses to take nalidixic acid, seem to show discrepancies after the Nielsen's test, howbeit the complete recuperation is self evident.

\section{REFERENCES}

1. Lee JW, Kim BJ, Kim MN. Photodynamic therapy: new treatment for recalcitrant Malassezia folliculitis. Lasers Surg Med. 2010;42:192-6.

2. Battersby AR, Fookes CJR, Matcham GWJ, McDonald E. Biosynthesis of the pigments of life: formation of the macrocycle. Nature. 1980;285:17-21.

3. Leeper FJ. The biosynthesis of porphyrins, chlorophylls, and vitamin B12. Natural Product Rep. 1983:2:19-47.

4. Layer G, Reichelt J, Jahn D. Structure and function of enzymes in heme biosynthesis. Protein Scien. 2010;19:1137-61.

5. Seinosuke U. Fungal glucoamylases and raw starch digestion. Trend Bioch Scien.1981;6:89-90.

6. Stollery N. Skin infections. Practitioner. 2014;258:32-3.

7. Bragg J, Pomeranz M. Papulopustular drug eruption due to an epidermal growth factor receptor inhibitors, erlotinib and cetuximab. Dermatol Online J. 2007;13:1.

8. Nielsen KP, Zhao L, Stamnesl JJ, Stamnes K, Moan J. The optics of human skin: Aspects important for human health: In: Solar Radiation and Human Health.Espen Bjertness Ed.2008: Oslo: The Norwegian Academy of Science and Letters.

9. Nichols EF. A study of the transmission spectra of certain substances in the infra-red. Phys Re. 1893;1:1-18.

Copyright by Lorenzo Martini. This is an open access article distributed under the terms of the Creative Commons Attribution License, which permits unrestricted use, distribution, and reproduction in any medium, provided the original author and source are credited.

Source of Support: Nil, Conflict of Interest: None declared. 